\title{
Sudden transition to chaos in plasma wave interactions
}

\author{
O. López-Rebollal, J. R. Sanmartín, ${ }^{\text {a) }}$ and E. del Río \\ Deptartmento, Física Aplicada, Escuela Técnica Superior de Ingenieros Aeronáuticos, \\ Universidad Politécnica de Madrid, Madrid, Spain
}

(Received 14 January 1998; accepted 7 May 1998)

\begin{abstract}
The coherent three-wave interaction, with linear growth in the higher frequency wave and damping in the two other waves, is reconsidered; for equal dampings, the resulting three-dimensional (3-D) flow of a relative phase and just two amplitudes behaved chaotically, no matter how small the growth of the unstable wave. The general case of different dampings is studied here to test whether, and how, that hard scenario for chaos is preserved in passing from 3-D to four-dimensional flows. It is found that the wave with higher damping is partially slaved to the other damped wave; this retains a feature of the original problem (an invariant surface that meets an unstable fixed point, at zero growth rate) that gave rise to the chaotic attractor and determined its structure, and suggests that the sudden transition to chaos should appear in more complex wave interactions. (C) 1998
\end{abstract} American Institute of Physics. [S1070-664X(98)02008-4]

\section{INTRODUCTION}

The coherent, quadratic interaction of three waves at or near resonance (3WRI) is an ubiquitous feature of weakly nonlinear mediums. The 3 WRI is especially important in plasmas, where dispersive effects can keep nonlinearities weak and electromagnetic waves make coupling to external energy sources easy. This applies to plasmas both unmagnetized (the case of stimulated Brillouin or Raman scattering) and magnetized (say, Alfvén or lower hybrid coupling). The 3 WRI has been extensively used to study the saturation of linear instabilities, or the inception of nonlinear instabilities in the case of waves with energies of different sign, and has been a basic nonlinear paradigm. ${ }^{1-3}$

For fully conservative modes with a small frequency mismatch $\nu$, the spaceindependent evolution can be obtained in terms of Jacobi elliptic functions if the three wave energies have equal signs. ${ }^{1,3}$ Solutions have been also found for linearly damped modes if all three dampings are equal $\left(\gamma_{1}\right.$ $=\gamma_{2}=\gamma_{3}$ ), the nonlinear coupling is lossless, and the mismatch vanishes. ${ }^{3}$ The case of both positive and negative energies is solved in terms of Weierstrass's elliptic function. ${ }^{4}$ The effect of equal dampings on the Weierstrass solution for the $\nu \neq 0$ interaction with negative energies has been studied recently. ${ }^{5}$

The 3WRI evolution for lossless coupling, when the higher frequency mode is linearly unstable (growth $\Gamma \equiv$ $\left.-\gamma_{1}>0\right)$ and the two other modes are equally damped $\left(\gamma_{2}\right.$ $=\gamma_{3}$ ), can be described by a three-dimensional (3-D) flow of two wave amplitudes and one relative phase. ${ }^{6-9}$ This reduced three-wave interaction can be simulated by two coupled oscillators with a near 2:1 frequency ratio. For $\Gamma$ $<\gamma_{2}$ the system is attracted to point sets of vanishing 3-D volume, and exhibits chaotic behavior for nonvanishing $\nu$, if not too large.

The above damped and driven near resonance was later

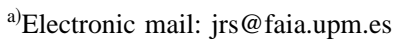

found to exhibit a sudden or hard transition to chaos: For very small $\Gamma$, a consistent analysis of the flow led to a onedimensional (1-D) chaotic map; ${ }^{10}$ a similar result was obtained for two oscillators at exact 1:1 resonance, under cubic coupling and linear driving with phase mismatch (a spherical swing). ${ }^{11}$ Actually, both results are particular examples of a general scenario for chaos; for $\Gamma$ positive, no matter how small, these systems present a fully developed chaotic attractor, which is absent at $\Gamma=0$. The limits $t \rightarrow+\infty$ and $\Gamma \rightarrow 0+$ do not commute because times on the attractor diverge as $\Gamma \rightarrow 0+$, which also makes an exact description of 3-D chaotic flows possible. ${ }^{12}$ Both the hard transition, and the effects of noise on it, have been experimentally verified by using two coupled electronic oscillators. ${ }^{13}$

The spatio-temporal evolution of fully conservative wave packets can be determined by means of an inverse scattering transform; this system has been found to support soliton solutions. ${ }^{14}$ The effect of damping and forcing on the $3 \mathrm{WRI}$ evolution has also been analyzed. Again, equal dampings were assumed for the stable wave packets, and the growth of the unstable wave included a diffusion-type term, to make the problem well posed (it provided a cutoff in wave number of the growth). ${ }^{15,16}$ Numerical studies for different parametric domains showed that the system may exhibit spatio-temporal chaos, which depends on the relative ordering of group velocities for the three modes.

There has been recent interest in more complex wave interactions. For evolution in time only, chaos was found in $\nu \neq 0$, conservative systems, for four-wave coupling (two triplets made of one electromagnetic, one ion-acoustic, and two Langmuir waves), ${ }^{17}$ and multiple-coupling of modes with different energy signs, in a plasma containing ion beams. ${ }^{18} \mathrm{~A}$ four-mode, cubic coupling, arising from the derivative nonlinear Schrödinger equation, was used to study the generation of damped Alfvén waves. ${ }^{19}$ Further studies, for evolution in time and space, concerned saturation and damping for five-wave interaction (two electromagnetic, two Langmuir, and one ion-acoustic wave packets), ${ }^{20}$ and five- 
wave coupling of gravity waves in deep water, for which the lowest order interaction involves four waves. ${ }^{21}$

In this paper we reconsider the hard transition to chaos for the general case of different dampings of the two stable waves $\left(\gamma_{3} \neq \gamma_{2}, \nu \neq 0, \Gamma \rightarrow 0+\right)$, thus keeping the interaction fully three-wave and the flow four dimensional. We want to ascertain whether, and how, the sudden setup of chaos, which seems to have a certain universal character, ${ }^{12}$ is retained in passing from 3-D to four dimensons (4-D). In Sec.II we recall the 3 WRI model. Sections III and IV deal with the attractor at $\Gamma=0$ and $\Gamma \rightarrow 0+$, respectively. Results are discussed in Sec.V.

\section{THEORETICAL MODEL}

For the coherent quadratic interaction of three modes with frequencies $\omega_{j}, j=1-3$, the equations describing the (space-independent) evolution of the corresponding complex amplitudes $a_{j}$ are $2,6,7,10$

$$
\begin{aligned}
& \dot{a}_{1}=\Gamma a_{1}-a_{2} a_{3} \exp (-i \nu t), \\
& \dot{a}_{2}=-\gamma_{2} a_{2}+a_{3}^{*} a_{1} \exp (i \nu t), \\
& \dot{a}_{3}=-\gamma_{3} a_{3}+a_{1} a_{2}^{*} \exp (i \nu t) .
\end{aligned}
$$

The nonlinear coupling was taken conservative; the case of a nonconservative coupling has been studied recently. ${ }^{22}$ Also, the frequency mismatch $\nu \equiv \omega_{1}-\omega_{2}-\omega_{3}$ (which may be taken positive without loss of generality); the linear dampings $\gamma_{2}$ and $\gamma_{3}$, and growth $\Gamma$; and the properly normalized amplitudes, were assumed small compared with the mode frequencies. Quite different models apply if the coherence is lost, leading to a random-phase approximation, ${ }^{23}$ or if nonlinearities are large. ${ }^{24}$

Writing $a_{j} \rightarrow a_{j} \exp \left(i \phi_{j}\right)$ above, with $a_{j}$ and $\phi_{j}$ now real and slowly time dependent, one obtains a fourth-order system of equations describing the slow evolution of $a_{1}, a_{2}, a_{3}$, and $\beta \equiv \frac{1}{2} \pi+\nu t+\phi_{1}-\phi_{2}-\phi_{3},{ }^{2,6,7}$

$$
\begin{aligned}
& \dot{a}_{1}=\Gamma a_{1}-a_{2} a_{3} \sin \beta, \\
& \dot{a}_{2}=-\gamma_{2} a_{2}+a_{1} a_{3} \sin \beta, \\
& \dot{a}_{3}=-\gamma_{3} a_{3}+a_{1} a_{2} \sin \beta, \\
& \dot{\beta}=\nu+\left(a_{1} \frac{a_{3}}{a_{2}}+a_{1} \frac{a_{2}}{a_{3}} \frac{a_{2} a_{3}}{a_{1}}\right) \cos \beta .
\end{aligned}
$$

Previous analyses ${ }^{6,7,10,12}$ considered the special case $\gamma_{3}$ $=\gamma_{2}$, Eqs. (1a) $-(1 \mathrm{~d})$ then yielding

$$
\frac{d}{d t}\left(a_{3}^{2}-a_{2}^{2}\right)=-2 \gamma_{2}\left(a_{3}^{2}-a_{2}^{2}\right) \text { for } \gamma_{3}=\gamma_{2} \text {. }
$$

Since the difference $a_{3}^{2}-a_{2}^{2}$ would now vanish exponentially with time, one could just take $a_{3}=a_{2}$ in studying the asymptotic behavior of Eqs. (1a)-(1d). The resulting thirdorder system also describes two coupled oscillators with a near 2:1 frequency ratio, and can be readily simulated in the laboratory; ${ }^{13}$ its conservative limit $\left(\Gamma=\gamma_{2}=\gamma_{3}=0\right)$ has been extensively studied. ${ }^{25}$ Here we consider the general case, with $\gamma_{3}>\gamma_{2}$ say, keeping a system of four equations.

Two immediate consequences of system (1a)-(1d) are

$$
\begin{aligned}
& \frac{\partial a_{1} \dot{a}_{1}}{a_{1} \partial a_{1}}+\frac{\partial a_{2} \dot{a}_{2}}{a_{2} \partial a_{2}}+\frac{\partial a_{3} \dot{a}_{3}}{a_{3} \partial a_{3}}+\frac{\partial \dot{\beta}}{\partial \beta}=2\left(\Gamma-\gamma_{2}-\gamma_{3}\right), \\
& \frac{d}{d t}\left(2 a_{1}^{2}+a_{2}^{2}+a_{3}^{2}\right)=4 \Gamma a_{1}^{2}-2 \gamma_{2} a_{2}^{2}-2 \gamma_{3} a_{3}^{2} .
\end{aligned}
$$

For $\Gamma<\gamma_{2}+\gamma_{3}$, as in the case of interest here $\left(\Gamma \rightarrow 0^{+}\right)$, the constant divergence of the flow, given by (2), is negative, the system thus being attracted to a point set of vanishing 4-D volume. For $\Gamma<0$, in particular, Eq. (3) shows that the trivial equilibrium state, $a_{1}=a_{2}=a_{3}=0$, is a global attractor. This equilibrium is unstable for $\Gamma>0$.

Next, note that the vector field defined by system (1a)(1d) is singular in the three-dimensional surface $a_{1}=0$, $d \beta / d t$ tending to $\infty$ as $a_{1} \rightarrow 0$ unless $a_{2} a_{3} \cos \beta$ vanishes with $a_{1}$. Since $d a_{j} / d t(j=1-3)$ keeps bounded in bounded domains, trajectories approaching that surface (except those corresponding to a null-measure set of initial conditions) become tangent to it. In what follows we will restrict attention to the region $a_{1}>0$. A similar argument applies for the surface $a_{3}=0$.

The case of surface $a_{2}=0$, where Eq. (1d) appears to be also singular, is somewhat different. In the following, we rewrite system (1a)-(1d) by introducing a new variable,

$$
r \equiv a_{3} / a_{2},
$$

to replace $a_{3}$. We then have

$$
\begin{aligned}
& \dot{a}_{1}=\Gamma a_{1}-r a_{2}^{2} \sin \beta, \\
& \dot{a}_{2}=-\gamma_{2} a_{2}+r a_{1} a_{2} \sin \beta, \\
& \dot{r}=-\left(\gamma_{3}-\gamma_{2}\right) r+\left(1-r^{2}\right) a_{1} \sin \beta, \\
& \dot{\beta}=\nu+\left(\frac{r^{2}+1}{r} a_{1}-r \frac{a_{2}^{2}}{a_{1}}\right) \cos \beta .
\end{aligned}
$$

It follows from (4c) that if a trajectory reaches the surface $r=1, r$ will remain less than unity thereafter. Equation (1d) is thus singular at $a_{3}=0$ [Eq. (4d) is singular at $r=0$ ], but not, in general, at $a_{2}=0$. Nonetheless, since $d a_{2} / d t$ vanishes with $a_{2}$ in (4b), trajectories approaching the surface $a_{2}=0$, which now appears invariant, become tangent to it too (even though $d \beta / d t$ is bounded).

\section{FLOW TOPOLOGY AT ZERO GROWTH RATE I}

For $\Gamma=0$ the full asymptotics of the system is readily determined. First, it now follows from Eq. (3) that $2 a_{1}^{2}+a_{2}^{2}$ $+a_{3}^{2}$ will keep diminishing as long as $a_{2}$ and $a_{3} \equiv r a_{2}$ are different from zero. This means that, for $\Gamma=0$, the entire flow is asymptotic to the 3 -D space $a_{2}=0$. Since this is an invariant surface, trajectories will be asymptotic to its critical elements with transverse stable manifolds.

Next, we consider the flow on $a_{2}=0$. For $\Gamma=0$ the system is described by

$$
\dot{a}_{1}=-r a_{2}^{2} \sin \beta
$$

together with Eqs. (4b)-(4d). Note that there is now a line of fixed points $\Lambda$ on $a_{2}=0$, given, in terms of the coordinate $r$, by 


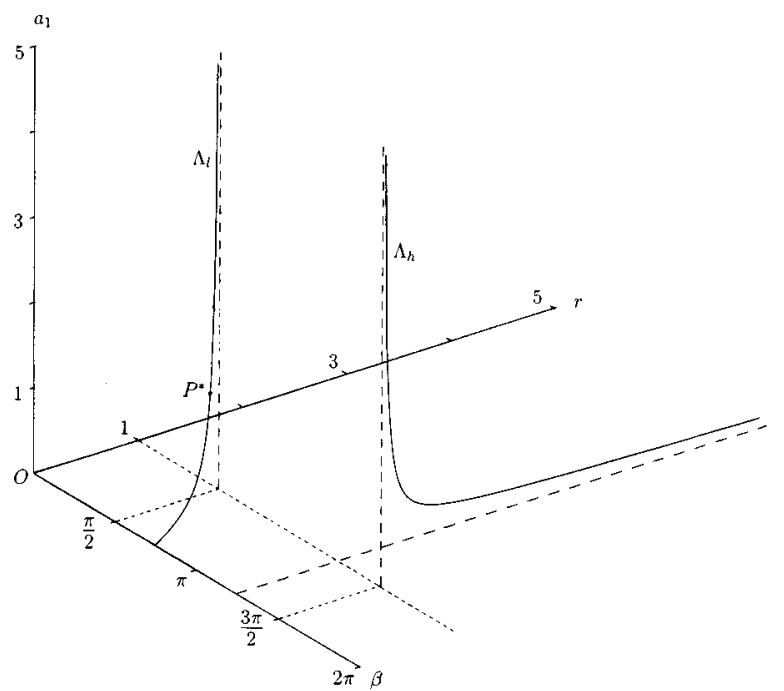

FIG. 1. Line of fixed points on the 3-D surface $a_{2}=0$, at $\Gamma=0 \quad\left(\gamma_{2}=1\right.$, $\left.\gamma_{3}=1.5, \nu=0.5\right)$. Branch $\Lambda_{h}$ is unstable on $a_{2}=0 ; \Lambda_{l}$ points above $P^{*}$ are unstable off that surface.

$$
\begin{aligned}
& a_{2}=0, \\
& a_{1}=\sqrt{\frac{\left(\gamma_{3}-\gamma_{2}\right)^{2} r^{2}}{\left(1-r^{2}\right)^{2}}+\frac{\nu^{2} r^{2}}{\left(1+r^{2}\right)^{2}},} \\
& \tan \beta=-\frac{\gamma_{3}-\gamma_{2}}{\nu} \frac{1+r^{2}}{1-r^{2}},
\end{aligned}
$$

with two branches, $\Lambda_{l}$ and $\Lambda_{h}$ (Fig. 1). Branch $\Lambda_{l}$ corresponds to values $r<1$ and $\pi / 2<\beta<-\tan ^{-1}\left(\gamma_{3}-\gamma_{2}\right) / \nu$ $<\pi$, and has the asymptote $r=1, \beta=\pi / 2$. Branch $\Lambda_{h}$ corresponds to values $r>1$ and $\pi<\tan ^{-1}\left(\gamma_{3}-\gamma_{2}\right) / \nu<\beta$ $<3 \pi / 2$, and has the asymptotes $r=1, \beta=3 \pi / 2$, and $a_{1}$ $=0, \beta=\tan ^{-1}\left(\gamma_{3}-\gamma_{2}\right) / \nu$.

To determine the stability of the fixed points we consider the eigenvalues of the linearized vector field in these points. Three eigenvalues have associated eigenvectors tangent to the invariant surface $a_{2}=0$ and are thus related to the stability of the flow in that surface; in terms of $r$ they are

$$
\lambda_{1}=0, \quad \lambda_{2,3}=\left(\gamma_{3}-\gamma_{2}\right) \frac{r^{2}+1}{r^{2}-1} \pm i \nu \frac{r^{2}-1}{r^{2}+1} .
$$

The null value of $\lambda_{1}$ is due to the very existence of line $\Lambda$, the corresponding eigenvector being tangent to the line itself. The eigenspace associated to eigenvalues $\lambda_{2}$ and $\lambda_{3}$ is tangent to the plane $a_{1}=$ constant at the respective fixed point; in fact, for the flow on $a_{2}=0, a_{1}$ keeps constant along trajectories, as shown by Eq. $\left(4 a^{\prime}\right)$. From the sign of the real part of $\lambda_{2,3}$ it follows that, for the flow on the invariant surface, points on the branch $\Lambda_{l}$ are stable and those on the branch $\Lambda_{h}$ are unstable.

The evolution of both $r$ and $\beta$ in each invariant plane $a_{1}=$ constant $\equiv a_{10}$, within the invariant space $a_{2}=0$, is given by Eqs. (4c) and (4d) particularized for the present case,

$$
\dot{r}=-\left(\gamma_{3}-\gamma_{2}\right) r+\left(1-r^{2}\right) a_{10} \sin \beta,
$$

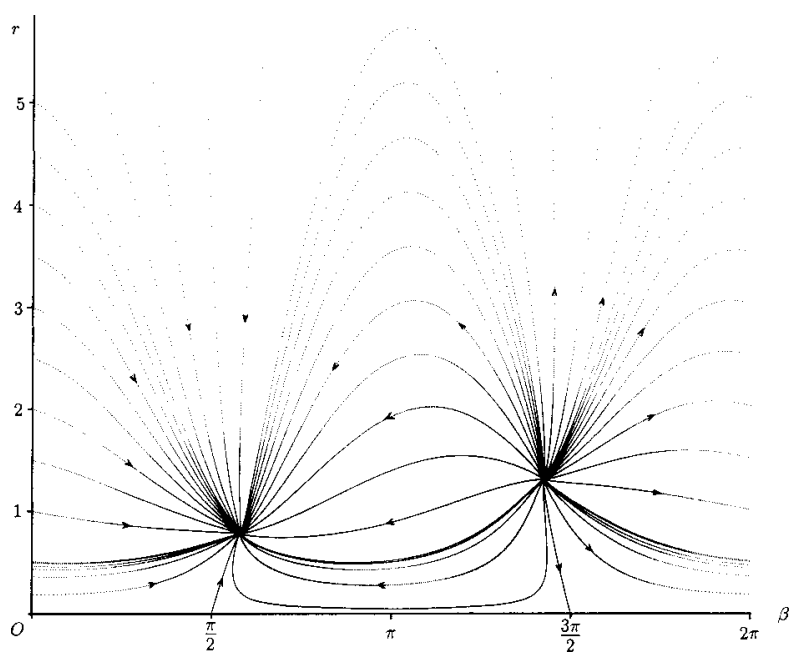

FIG. 2. Flow in a plane $a_{1}=$ constant $\equiv a_{10}$ on $a_{2}=0$, at $\Gamma=0 ; \gamma_{2}, \gamma_{3}$, and $\nu$ as in Fig. 1, $a_{10}=1$. The fixed points (foci) are the intersections of the plane with branches $\Lambda_{l}(r<1)$ and $\Lambda_{h}(r>1)$.

$$
\dot{\beta}=\nu+a_{10} \frac{r^{2}+1}{r} \cos \beta .
$$

At large $r$, Eqs. (6a) and (6b) have the family of trajectories $r \approx \pm r_{\max } \cos \beta$ (Fig. 2), with $r$ maximum at $\beta=0$ or $\pi$, and $d r / d t>0(<0)$ for $\beta>\pi(<\pi)$. There are two separatrices,

$$
\frac{\nu}{2 a_{10} r} \approx \beta-\frac{\pi}{2} \quad \text { or } \quad \frac{3}{2} \pi-\beta \quad(r \gg 1)
$$

coming from, and going to, infinity at the limit angles $\beta$ $=\pi / 2$ and $3 \pi / 2$, respectively; they start or end at fixed points that are intersections of branches $\Lambda_{l}$ and $\Lambda_{h}$ with the $a_{10}$ plane [two-dimensional (2-D) foci with eigenvalues $\left.\lambda_{2,3}\right]$. Figure 2 also shows how the trajectories, once having reached the value $r=1$, remain below this line, as advanced in Sec. II. At small $r$ the flow becomes tangent to the line $r=0$, and is described by the family $r \cos \beta \approx \pm r_{\min }$, with $r$ minimum at $\beta=0$ or $\pi$, and $d r / d t>0(<0)$ for $\beta<\pi$ $(>\pi)$; again, there are two separatrices,

$$
\frac{\nu r}{2 a_{10}} \approx \beta-\frac{\pi}{2} \quad \text { or } \quad \frac{3}{2} \pi-\beta, \quad(r \ll 1)
$$

leaving, and reaching, the line $r=0$ at the angles $\beta=\pi / 2$ and $3 \pi / 2$. Except for the separatrices leaving the $r>1$ unstable focus toward the limit angle $\beta=3 \pi / 2$, the entire flow tends to the stable focus at $r<1$; in the space $a_{2}=0$, the entire flow moves from the unstable branch $\Lambda_{h}$ to the stable branch $\Lambda_{l}$.

We now turn our attention to the stability of $\Lambda$ points off the surface $a_{2}=0$. The corresponding eigenvalue is clearly the factor multiplying $a_{2}$ in Eq. (4b), $\lambda_{4}=-\gamma_{2}+r a_{1} \sin \beta$, which, in terms of the $r$ coordinate, reads

$$
\lambda_{4}=\frac{\gamma_{3} r^{2}-\gamma_{2}}{1-r^{2}} .
$$


The associated eigenvector is transverse to the surface $a_{2}$ $=0$; it is in fact parallel to the $a_{2}$ axis. For motion off that surface, all points on the $\Lambda_{h}$ branch are stable, whereas only those points on the $\Lambda_{l}$ branch with

$$
r<r_{P *} \equiv \sqrt{\gamma_{2} / \gamma_{3}}
$$

are stable. Hence, for the flow in the entire four-dimensional space, the stable fixed points of $\Lambda$ are those on the $r<1$ branch satisfying condition (7). For the point $P^{*}$ separating stable from unstable points on $\Lambda_{l}$, we have

$$
\begin{aligned}
& a_{1 P^{*}}=\frac{\sqrt{\gamma_{2} \gamma_{3}}}{\sin \beta_{P^{*}}}, \\
& a_{2 P^{*}}=0, \\
& r_{P^{*}}=\sqrt{\frac{\gamma_{2}}{\gamma_{3}}}, \\
& \tan \beta_{P^{*}}=-\frac{\gamma_{2}+\gamma_{3}}{\nu} \quad\left(\pi / 2<\beta_{P^{*}}<\pi\right) .
\end{aligned}
$$

We may then conclude that, for $\Gamma=0$, the attractor of the flow is the $\Lambda_{l}$ arc below $P^{*}$ in Fig. 1 (a point set of zero $4-\mathrm{D}$ volume on the surface $a_{2}=0$ ). For generic initial conditions, trajectories approach and flow tangent to $a_{2}=0$, and cross the surface $r=1$, ending on $\Lambda_{l}$, below $P^{*}$, or approaching $\Lambda_{l}$, above. Note that $\Lambda_{l}$ points above $P^{*}$ in Fig. 1 have a 1-D unstable manifold transverse to $a_{2}=0$, corresponding to the positive sign of the eigenvalue $\lambda_{4}$. There are thus orbits that leave that surface at those points and end on the $\Lambda_{l}$ points below $P^{*}$, all of which have stable manifolds transverse to $a_{2}=0$ (and lie in the $r<1$ domain). Since these heteroclinic orbits are unstable manifolds of some fixed points, they have a local attractive character for the trajectories approaching the $\Lambda_{l}$ arc below $P^{*}$, from above. As we shall see in Sec. IV, the line branch $\Lambda_{l}$ and the family of heteroclinic orbits connecting points on $\Lambda_{l}$ are the topological elements that compose the asymptotic behavior of the flow for $\Gamma \rightarrow 0+$.

\section{TRANSITION TO CHAOS AT $\Gamma \rightarrow 0+$}

For $\Gamma$ positive, Eqs. (4a)-(4d) have no line of fixed points, just one fixed point, $P$,

$$
\begin{aligned}
& a_{1 P}=\frac{\sqrt{\gamma_{2} \gamma_{3}}}{\sin \beta_{P}}, \quad a_{2 P}=\frac{\sqrt{\Gamma \gamma_{3}}}{\sin \beta_{P}}, \quad r_{P}=\sqrt{\frac{\gamma_{2}}{\gamma_{3}}}, \\
& \tan \beta_{P}=\frac{\Gamma-\gamma_{2}-\gamma_{3}}{\nu} .
\end{aligned}
$$

As $\Gamma$ is decreased to zero, $P$ tends to the point $P^{*}$ on $\Lambda_{l}$. Note that $P$ does not exist for negative $\Gamma$.

The characteristic, quartic equation for the eigenvalues of this fixed point depends on the ratios

$$
\bar{\Gamma} \equiv \Gamma / \gamma_{2}, \quad \bar{\gamma} \equiv\left(\gamma_{3}-\gamma_{2}\right) / \gamma_{2}, \quad \bar{\nu} \equiv \nu / \gamma_{2} .
$$

In this three-dimensional parametric space, the domain of stability for $P$ may be shown to be bounded by the surface

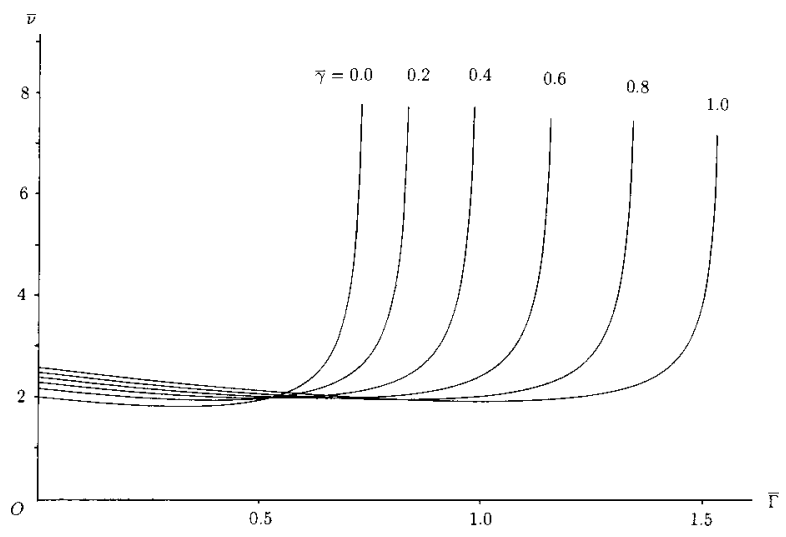

FIG. 3. Surface $\bar{\nu}(\bar{\Gamma}, \bar{\gamma})$ for Hopf bifurcation of fixed point $P$, which is unstable below the surface; $\bar{\Gamma} \equiv \Gamma / \gamma_{2}, \bar{\gamma} \equiv\left(\gamma_{3}-\gamma_{2}\right) / \gamma_{2}, \bar{\nu} \equiv \nu / \gamma_{2}\left(P \rightarrow P^{*}\right.$ as $\Gamma \rightarrow 0)$.

$$
\bar{\nu}=\sqrt{\frac{-c_{1}+\sqrt{c_{1}^{2}+4 c_{2} c_{0}}}{2 c_{2}}},
$$

where $c_{0}, c_{1}$, and $c_{2}$ are functions of $\bar{\Gamma}$ and $\bar{\gamma}$,

$$
\begin{aligned}
c_{0}= & \bar{\gamma}^{4}+7 \bar{\gamma}^{3}+18 \bar{\gamma}^{2}+20 \bar{\gamma}+8-\left(3 \bar{\gamma}^{3}+13 \bar{\gamma}^{2}+20 \bar{\gamma}+10\right) \bar{\Gamma} \\
& +\left(3 \bar{\gamma}^{2}+9 \bar{\gamma}+6\right) \bar{\Gamma}^{2}-(\bar{\gamma}+1) \bar{\Gamma}^{3}, \\
c_{1}= & 2\left[\bar{\gamma}^{4}+5 \bar{\gamma}^{3}+10 \bar{\gamma}^{2}+10 \bar{\gamma}+4-\left(\bar{\gamma}^{3}+9 \bar{\gamma}^{2}+16 \bar{\gamma}+8\right) \bar{\Gamma}\right. \\
& \left.+\left(\bar{\gamma}^{2}+3 \bar{\gamma}+2\right) \bar{\Gamma}^{2}+(\bar{\gamma}-1) \bar{\Gamma}^{3}\right] /(\bar{\gamma}+2-\bar{\Gamma})^{2}, \\
c_{2}= & 3(\bar{\gamma}+1) \frac{\bar{\gamma}^{3}+2 \bar{\gamma}^{2}+\left(\bar{\gamma}^{2}+2 \bar{\gamma}+2\right) \bar{\Gamma}-(\bar{\gamma}+2) \Gamma^{2}-\bar{\Gamma}^{3}}{(\bar{\gamma}+2-\bar{\Gamma})^{4}} .
\end{aligned}
$$

Figure 3 shows this surface, with $P$ being stable above it. When $\bar{\nu}$ drops below the surface in Fig. 3 , in the transition to instability, a periodic orbit is born through a Hopf bifurcation. As $\bar{\nu}$ is decreased further, that orbit loses stability to a new orbit of double period. Numerical integration of system (4a)-(4d) shows that a period-doubling cascade leads to the appearance of chaotic attractors.

Here we are only interested in the limit behavior at $\Gamma$ $\rightarrow 0+$. We find that a Hopf bifurcation, followed by a period-doubling cascade, occurs at positive $\Gamma$ no matter how small. This suggests that for $\bar{\nu}$ fixed and low enough, a sudden transition to chaos sets up when $\Gamma$ changes from negative to positive values, with a fully developed chaotic attractor existing at positive $\Gamma$ no matter how small, and $a_{2}$ and $a_{3}$, in particular, going through an unending sequence of drops and rises. This entails no contradiction with Eq. (3). It comes out that, for $\Gamma \rightarrow 0+$, the system lounges in the neighborhood of $\Lambda_{l}$ for a time of order $1 / \Gamma \rightarrow+\infty$, with $a_{2}$ and $r a_{2}$ exponentially small for most of that time, and $2 a_{1}^{2}+a_{2}^{2}$ $+a_{3}^{2} \approx 2 a_{1}^{2}$ rising in Eq. (3) at a vanishing rate; Eq. (4a) then reads $\dot{a}_{1} \approx \Gamma a_{1}$.

First, note that, as found in Sec. III, the eigenvalues for the characteristic equation at $P^{*}$ are $\lambda_{1}=\lambda_{4}=0, \lambda_{2,3}=$ $-\left(\gamma_{3}-\gamma_{2}\right) \pm i \nu\left(\gamma_{2}-\gamma_{3}\right) /\left(\gamma_{2}+\gamma_{3}\right)$. Two eigenvalues for $P$ will thus vanish with $\Gamma$, the corresponding eigenvectors 

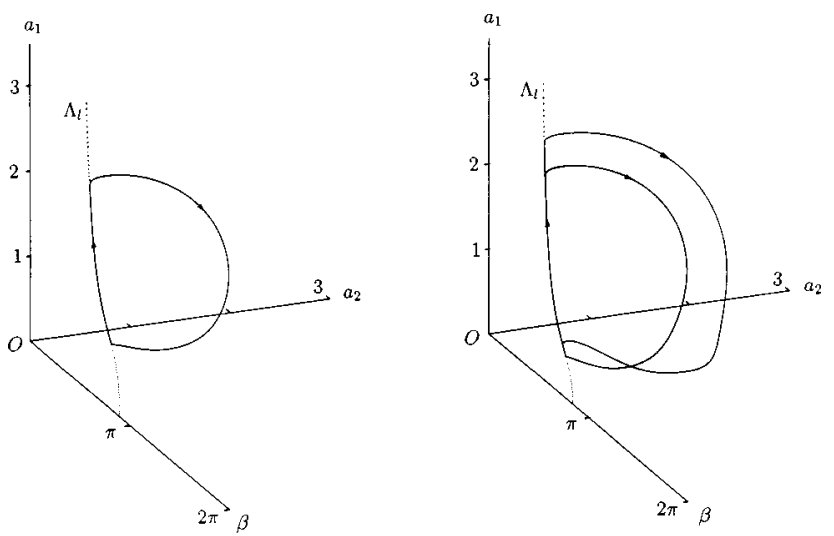

FIG. 4. Projection on space $a_{1}, a_{2}, \beta$ of stable periodic orbits for $\Gamma=0.01$, $\gamma_{2}=1, \gamma_{3}=1.5$, and (a) $\nu=1.5$, (b) $\nu=1$.

being tangent to $\Lambda_{l}$ and transverse to $a_{2}=0$, respectively. For small $\Gamma, \lambda_{1}$ and $\lambda_{4}$ are approximately determined by a quadratic equation,

$$
\lambda^{2}+b \Gamma+c \lambda^{3}+d \Gamma \lambda \approx 0, \quad(\Gamma \text { small }),
$$

where $a, b$, and $c$ are (positive) functions of $\bar{\nu}$ and $\bar{\gamma}$. We thus find

$$
\lambda_{1,4} \approx \pm i_{\sqrt{ }} b \sqrt{ } \Gamma+\frac{1}{2}(b c-d) \Gamma,
$$

with a Hopf bifurcation occurring for $b c-d=0$, which reads

$$
3\left(\frac{\bar{\gamma}}{2+\bar{\gamma}}\right)^{2} \bar{\nu}^{4}+2\left(2+2 \bar{\gamma}+\bar{\gamma}^{2}\right) \bar{\nu}^{2}-(2+\bar{\gamma})^{4}=0 ;
$$

this recovers Eq. (9) with $\Gamma=0$ in $c_{0}, c_{1}$, and $c_{2}$.

Next, consider numerical evidence such as given in Figs. 4(a), 4(b) and 5, for a single trajectory each. Figure 4(a), 4(b), and 5 show two stable periodic orbits and an apparently chaotic attractor, projected in the 3-D space $a_{1}, a_{2}$, and $\beta$, for $\bar{\Gamma}=0.01, \bar{\gamma}=0.5$, and three decreasing values of $\bar{\nu}$ be-

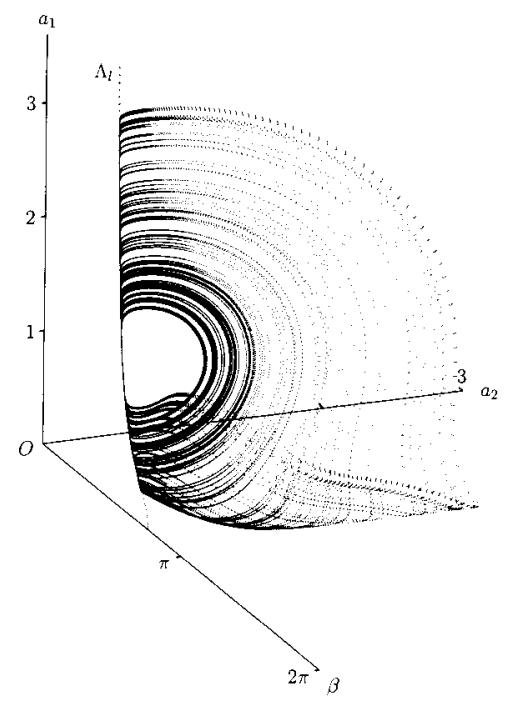

FIG. 5. Projection on space $a_{1}, a_{2}, \beta$ of a chaotic attractor (obtained from integrating a single trajectory); $\Gamma, \gamma_{2}$, and $\gamma_{3}$ as in Figs. 4(a) and 4(b) $\nu=0.55$.

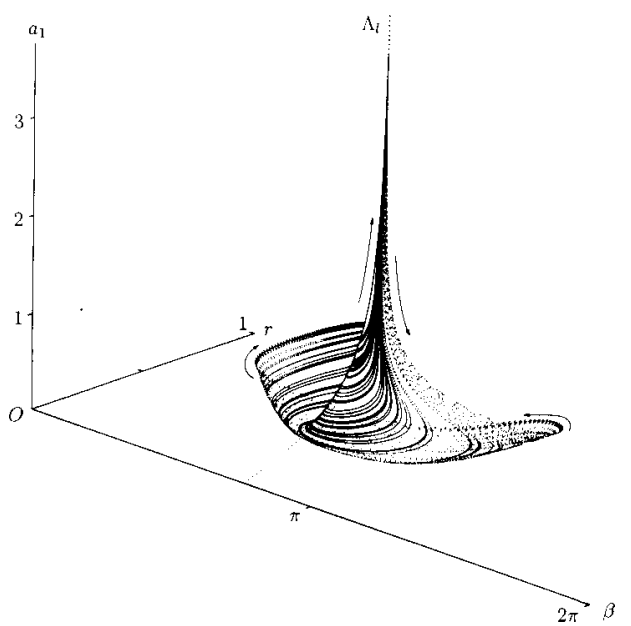

FIG. 6. Projection on space $a_{1}, r, \beta$ of the trajectory in Fig. 5.

yond the Hopf bifurcation. The trajectories form loops that move away from the plane $a_{2}=0$ and approach it again; this stage is followed by a process during which $a_{1}$ increases until a new loop is formed. The $a_{1}$ rise appears to occur along the $(\Gamma=0) \Lambda_{l}$ branch, and the loops to follow heteroclinic orbits of the $\Gamma=0$ family previously mentioned. Figure 6 shows the attractor of Fig. 5 projected into the space $a_{1}, r, \beta$.

To understand this behavior, note that, as previously seen, trajectories for $\Gamma=0$ tend to points on $\Lambda_{l}$ below $P^{*}$, getting close to the heteroclinic orbits in the neighborhood of the surface $a_{2}=0$. Let us assume that, once transients have died out, the flow for very small $\Gamma$ follows closely an heteroclinic orbit (corresponding to some $\Lambda_{l}$ point $M$ above $\left.P^{*}\right)$ in the approach to a point $m$ below $P^{*}$, on $a_{2}=0$. Now, however, because of the term $\Gamma a_{1}$ in Eq. (4a), $a_{1}$ should, at some point, start growing at the vanishing rate $\Gamma$, keeping close to $\Lambda_{l}$, with Eqs. (4c) and (4d) reading

$$
\begin{aligned}
& \dot{r}=-\left(\gamma_{3}-\gamma_{2}\right) r+\left(1-r^{2}\right) a_{1} \sin \beta=O(\Gamma), \\
& \dot{\beta}=\nu+\frac{r^{2}+1}{r} a_{1} \cos \beta=O(\Gamma) .
\end{aligned}
$$

Also, in terms of the eigenvalue $\lambda_{4}$ of Sec. III, Eq. (4b) can be written as $a d_{2} / d t=\lambda_{4} a_{2}$; since $\lambda_{4}$ is negative for $\Lambda_{l}$ points below $P^{\prime}$ (and positive above $P^{*}$ ), and the $a_{1}$ rise takes times of order $1 / \Gamma, a_{2}$ will be exponentially small $\left(-\ln a_{2} \sim 1 / \Gamma\right)$ throughout it. Also, $a_{2}$ will start growing once $P^{*}$ is reached; when values $a_{2} \sim{ }_{\sqrt{ }} \Gamma$ are attained, $a_{1}$ finally reaches a maximum, and the trajectory starts separating from both $\Lambda_{l}$ and the surface $a_{2}=0$, following another heteroclinic orbit that returns to $\Lambda_{l}$ again. A detailed discussion of the time sequence on a similar though simpler attractor can be found in Ref. 11 .

The $\Gamma \rightarrow 0+$ attractor may now be described by an exact 1-D map of maxima. If one represents every maximum of $a_{1}$ $\left(a_{1 M^{\prime}}\right)$ for the trajectory in Figs. 5 and 6 as function of the preceding maximum $\left(a_{1 M}\right)$ one obtains the point set of Fig. 7. As $\Gamma$ is decreased, that set approaches the continuous curve also shown in Fig. 7. From the above discussion, this limit curve can be determined by a two-step algorithm. In the 


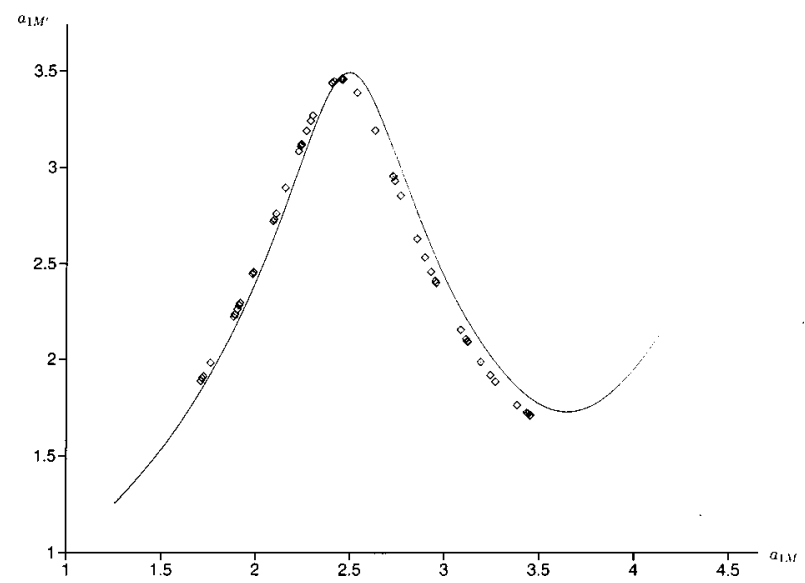

FIG. 7. One-dimensional map of $a_{1}$ maxima for the trajectory in Figs. 5 and 6 (points) and for the $\Gamma=0$, limit attractor at the values $\gamma_{2}, \gamma_{3}$ and $\nu$ of that trajectory (continuous line).

first step, one numerically follows the heteroclinic orbit from any point $M$ on $\Lambda_{l}$ above $P^{*}$, to the corresponding point $m$ below $P^{*}$. The second step is the rise along $\Lambda_{l}$ at vanishing rate $(\Gamma \rightarrow 0, \Gamma t \sim 1)$ up to the next maximum $M^{\prime}$. To analytically determine this second step note that, no matter how close the solution to a heteroclinic $M \rightarrow m$ orbit, Eq. (4a) will ultimately read $d a_{1} / d t=\Gamma a_{1}$ and Eqs. (4c) and (4d) will take the form $\left(4 \mathrm{c}^{\prime}\right),\left(4 \mathrm{~d}^{\prime}\right)$. Using also $d a_{2} / d t=\lambda_{4} a_{2}$, one obtains

$$
\frac{-\gamma_{2}+r a_{1} \sin \beta}{a_{1}} d a_{1}=\Gamma d \ln a_{2},
$$

where all coordinates on the left-hand side can be rewritten in terms of one of them, say $r$, by using Eqs. (5b) and (5c) for the line $\Lambda$.

The integral of the right-hand side above, for the entire rise from $m$ to $M^{\prime}$ (with $\ln \left(1 / a_{2}\right)$ small compared with $1 / \Gamma$ at either end), will vanish in the limit $\Gamma \rightarrow 0+$. We thus find

$$
\begin{aligned}
\int_{r_{m}}^{r_{M^{\prime}}-\gamma_{2}+r a_{1} \sin \beta} & a_{1} \\
a_{1} & =O\left(\Gamma \ln \frac{1}{\Gamma}\right) \rightarrow 0 \\
& \rightarrow F\left(r_{M^{\prime}} ; \bar{\gamma}, \bar{\nu}\right)=F\left(r_{m} ; \bar{\gamma}, \bar{\nu}\right)
\end{aligned}
$$

with $F$ defined as

$$
\begin{aligned}
F(r ; \bar{\gamma}, \bar{\nu}) \equiv & \frac{2 \bar{\gamma}}{1-r^{2}}-\ln r^{2}-\bar{\nu} \tan ^{-1}\left(\frac{\bar{\gamma}}{\bar{\nu}} \frac{1+r^{2}}{1-r^{2}}\right) \\
& -\left(1+\frac{\bar{\gamma}}{2}\right) \ln \left[\frac{\bar{\gamma}^{2}}{\left(1-r^{2}\right)^{2}}+\frac{\bar{\nu}^{2}}{\left(1+r^{2}\right)^{2}}\right] .
\end{aligned}
$$

Note that $F$ has a single minimum at $P^{*}$; also, $F \rightarrow+\infty$ at both ends of the $r$ range for $\Lambda_{l}(r=0,1)$. Hence, for any value $r_{m}<r_{P^{*}}$, Eq. (10) determines a value $r_{M^{\prime}}>r_{P *}$. Through Eqs. (5b) and (5c) one can then establish the relation between $a_{1 m}$ and $a_{1 M^{\prime}}$ that leads to the limit map in Fig. 7.

To understand why this map exhibits the critical points that give rise to its chaotic behavior, note, first, that the lower $m$ lies on $\Lambda_{l}$ in Fig. 1, the higher is $M^{\prime}\left(d a_{1 M^{\prime}} / d a_{1 m}<0\right)$, as follows from Eq. (10). Next, consider the $\Gamma=0$ heteroclinic orbit for $M$ close to $P^{*}$. Since both $M$ and $m$ lie on $\Lambda_{l}$ and are close to each other, we will have $\beta<\pi$, and $a_{1}$ decreasing in $\left(4 \mathrm{a}^{\prime}\right)$, throughout that orbit. Thus, $a_{1 m}$ will drop when $a_{1 M}$ is raised, leading to $d a_{1 M^{\prime}} / d a_{1 M}>0$; this is the behavior to the left of the maximum in Fig. 7.

The maximum of $\beta$ in the heteroclinic orbit increases, however, with $a_{1 M}$, and reaches $\pi$ for the abscissa corresponding to the maximum in Fig. 7. For higher $a_{1 M}$ the orbit makes a excursion into the $\beta>\pi$ domain, with $a_{1}$ increasing in Eq. $\left(4 \mathrm{a}^{\prime}\right)$, before finally reaching $m$ at a $\beta<\pi$ value. This results in $a_{1 m}$ increasing with $a_{1 M}$, and a negative value for $d a_{1 M^{\prime}} / d a_{1 M}$, as in the map to the right of the maximum. As $a_{1 M}$ is raised further, $m$ reaches $P^{*}$; this occurs for the abscissa $a_{1 M}$ corresponding to the minimum in Fig. 7. For higher $a_{1 M}$, the heteroclinic orbit approaches a point on $\Lambda_{l}$ above $P^{*}$ and then turns around to end at $m$, below, and close to, $P^{*}$. Now $m$ drops with increasing $a_{1 M}$, leading again to $d a_{1 M^{\prime}} / d a_{1 M}>0$, as in the map to the right of the minimum. We note that, for certain $\bar{\gamma}, \bar{\nu}$ domain, when $a_{1 M}$ reaches high enough in the attractor, the map may actually exhibit multiple maxima and minima.

Note finally that Eqs. (5a)-(5c) and (11), in the limit $\gamma_{3}-\gamma_{2} \rightarrow 0$, recover known results for the special case $\gamma_{3}=\gamma_{2}{ }^{12}$ In Eqs. $(5 a)-(5 c)$ the entire line $\Lambda$ collapses into the plane $r=1$ and, using (5c) in (5b), becomes $a_{2}=0$, $r=1,2 a_{1} \cos \beta+\nu=0$. The branches $\Lambda_{l}$ and $\Lambda_{h}$ now join at $\beta=\pi$, where $a_{1}$ has a minimum, $\nu / 2$. Equation (11) itself becomes

$$
\begin{aligned}
F\left(a_{1} / \gamma_{2} ; \nu / \gamma_{2}\right) \equiv & \sqrt{\frac{4 a_{1}^{2}-\nu^{2}}{\gamma_{2}^{2}}} \\
& -\frac{\nu}{\gamma_{2}} \tan ^{-1} \sqrt{\frac{4 a_{1}^{2}-\nu^{2}}{\nu^{2}}}-2 \ln \frac{a_{1}}{\gamma_{2}} .
\end{aligned}
$$

\section{DISCUSSION OF RESULTS}

We have studied a sudden transition to chaos in 3WRI, with lossless coupling, frequency mismatch, and linear dampings $\gamma_{1} \equiv-\Gamma, \gamma_{2}$, and $\gamma_{3}\left(>\gamma_{2}\right)$. The resulting 4-D flow for amplitudes $a_{1}, a_{2}$, and $a_{3}$, and a relative phase, has (i) an invariant 3-D surface $\left(a_{2}=0\right)$, (ii) a rest state that is unstable for $\Gamma>0$, and (iii) a second fixed point, for $\Gamma \geqslant 0$. At $\Gamma=0$, this point lands at $P^{*}$ on the invariant surface, where it generates a line $\Lambda$ of fixed points with two disjoint branches. $P^{*}$ lies on the branch $\left(\Lambda_{l}\right)$ stable for motion on $a_{2}=0$ and is doubly degenerate, separating $\Lambda_{l}$ arcs stable (at low $a_{1}$ ) and unstable (at high $a_{1}$ ) for motion off $a_{2}=0$; heteroclinic orbits off $a_{2}=0$ join both arcs. The attractor is the rest state for negative $\Gamma$ (all three waves damped), and the $\Lambda_{l}$ arc below $P^{*}$ for $\Gamma=0$.

For $\Gamma \rightarrow 0+$ and a small enough mismatch, the system has a fully developed chaotic attractor that may be described by a noninvertible, 1 -D limit map of $a_{1}$ maxima. Each iteration in the map is made up of a rise up $\Lambda_{l}$, from below to above $P^{*}$, on $a_{2}=0$, and a heteroclinic orbit off $a_{2}=0$ that returns to $\Lambda_{l}$ below $P^{*}$. The $\Gamma \rightarrow 0+$ limit of long-time solutions is thus singular: it does not exist at $\Gamma=0$ (the limits 
$t \rightarrow+\infty$ and $\Gamma \rightarrow 0+$ do not commute, the rise up $\Lambda_{l}$ in the singular solution taking a time $\sim 1 / \Gamma$ ). As a result, the map is exactly 1-D (the Cantor structure preserving determinism in the reduced 1-D description vanishes with $\Gamma$ ), and exhibits multiple critical points because the heteroclinic orbits map different $\Lambda_{l}$ segments above $P^{*}$ into a common segment below $P^{*}$.

Essential to the transition is the fact that $d\left(a_{3} / a_{2}\right) / d t$ is negative at $a_{3} / a_{2}=1$. This makes the surface $a_{2}=0$ effectively invariant; in the reduced $3 \mathrm{WRI}\left(\gamma_{3}=\gamma_{2}\right)$ case that surface was invariant from the outset. As a minor difference, there existed at $\Gamma=0$, in the reduced case, a second invariant surface intersecting $a_{2}=0$ at $\Lambda$, and allowing an analytical determination of the limit 1-D map; here the heteroclinic orbits are determined numerically. Our results suggest that the transition should set up in quite general interactions, e.g., those involving multiple waves, with the excited and less damped waves playing the fundamental role. This is important because certain features of the transition might make it hard to detect.

The attractor of our system has zero $a_{2}$ (and $a_{3}$ ) for $\Gamma$ $\leqslant 0$, and has $a_{2}$ small for small, positive $\Gamma$ if the frequency mismatch is above the surface in Fig. $3\left(a_{2 P} / a_{1 P}\right.$ $\left.=\sqrt{\Gamma / \gamma_{2}}\right)$. On the other hand, for $\Gamma$ small and mismatch below that surface, the ratio $a_{2} / a_{1}$ for either limit cycle or chaotic attractor moves repeatedly from almost vanishing values on $\Lambda_{l}$ to values of order unity in the heteroclinic orbits. This sudden break in $a_{2}$ behavior would be the easiest feature to detect in the transition. However, the long rise up $\Lambda_{l}(t \sim 1 / \Gamma)$ might allow effects ignored in the model to distort or hide the transition. In a recent experimental verification of the reduced case $\left(\gamma_{3}=\gamma_{2}\right)$ by using two coupled electronic oscillators, noise distorted the 1-D map when $\Gamma$ was very small and $a_{2}$, which becomes exponentially small in $1 / \Gamma$ during the $\Lambda_{l}$ rise, dropped below the noise level. ${ }^{13}$ This could also occur for very small frequency mismatch (note that the attractor is chaotic for vanishing mismatch in Fig. 3).

Controlling the growth $\Gamma$ of a typical plasma instability (due to a beam interaction ${ }^{18}$ or any negative Landau damping) in order to verify the transition might be hard. The simplest scenario would have the wave $l$ in our interaction triplet, made unstable by a pump coupled in a second triplet. This might involve five waves (two electromagnetic, two Langmuir, and one ion acoustic) ${ }^{20}$ or four waves (one whistler, two lower hybrid and one ion-acoustic). ${ }^{26}$ Our uniform wave-train model should be well suited to the study of the transition. A proper description of wave envelopes dependent on both time and space $(x)$ requires setting $\Gamma \rightarrow \Gamma$ $-D \partial^{2} / \partial x^{2}$; the last term provides a cutoff in wave number of the growth. ${ }^{15,16}$ For $D$ large enough, or $\Gamma$ small enough as here, the space dependence might be ignorable.

Note added in proof. When this paper was being set in print, it came to our attention the paper "Non-symmetrical three-wave resonance," in Wave Motion 20, 201 (1994) by D. W. Hughes and M. R. E. Proctor, which deals with our same problem.

\section{ACKNOWLEDGMENTS}

This work was supported by the Comision Interministerial de Ciencia y Tecnología of Spain under Grant No. PB940417-C03-01.

${ }^{1}$ R. C. Davidson, Methods in Nonlinear Plasma Theory (Academic, New York, 1972), Chap. 6.

${ }^{2}$ A. Bers, in Plasma Physics-Les Houches 1972, edited by C. De Witt and J. Peyreaud (Gordon and Breach, New York, 1975), pp. 117-215.

${ }^{3} \mathrm{~J}$. Weiland and $\mathrm{H}$. Wilhelmsson, Coherent Nonlinear Interaction of Waves and Plasmas (Pergamon, New York, 1977).

${ }^{4}$ D. Pfirsch, Z. Naturforsch. Teil A 45, 839 (1990).

${ }^{5}$ D. Y. Li, D. Y. Mao, and D. Y. Wang, J. Plasma Phys. 57, 861 (1997).

${ }^{6}$ S. Y. Vyshkind and M. I. Rabinovich, Sov. Phys. JETP 44, 292 (1976).

${ }^{7}$ J. M. Wersinger, J. M. Finn, and E. Ott, Phys. Rev. Lett. 44, 453 (1980); Phys. Fluids 23, 1142 (1980).

${ }^{8}$ C. Meunier, M. N. Bussac, and G. Laval, Physica D 4, 236 (1982).

${ }^{9}$ M. N. Bussac, Phys. Rev. Lett. 49, 1939 (1982).

${ }^{10}$ D. W. Hughes and M. R. E. Proctor, Physica D 46, 163 (1990).

${ }^{11}$ J. R. Sanmartín, O. López-Rebollal, and N. de Paola, Physica D 69, 148 (1993)

${ }^{12}$ O. López-Rebollal and J. R. Sanmartín, Physica D 89, 204 (1995).

${ }^{13}$ E. del Río, J. R. Sanmartín, and O. López-Rebollal, "Experimental evidence of a hard transition to chaos," to appear in Int. J. Bifurcation Chaos Appl. Sci. Eng.

${ }^{14}$ D. J. Kaup, A. Reiman, and A. Bers, Rev. Mod. Phys. 51, 275 (1979).

${ }^{15}$ C. C. Chow, A. Bers, and A. K. Ram, Plasma Phys. Controlled Fusion 34, 1945 (1992).

${ }^{16}$ C. C. Chow, Physica D 81, 237 (1995).

${ }^{17}$ A. C. L. Chian, S. R. Lopes, and J. R. Abaldo, Physica D 99, 269 (1996).

${ }^{18}$ C. S. Kueny and P. J. Morrison, Phys. Plasmas 2, 1926 (1995); 2, 4149 (1995).

${ }^{19}$ S. Rauf and J. A. Tataronis, J. Plasma Phys. 55, 173 (1996).

${ }^{20}$ T. Kolber, W. Rozmus, and V. T. Tikhonchuk, Phys. Fluids B 5, 138 (1993).

${ }^{21}$ A. I. Dyachenko, Y. V. Lvov, and V. E. Zakharov, Physica D 87, 233 (1995).

${ }^{22}$ D. W. Hugues and M. R. E. Proctor, J. Fluid Mech. 244, 583 (1992).

${ }^{23}$ P. Terry and W. Horton, Phys. Fluids 25, 491 (1982).

${ }^{24}$ G. I. de Oliveira, L. P. L. de Oliveira, and F. B. Rizzato, Physica D 104, 119 (1997).

${ }^{25}$ E. Breitenberger and R. D. Mueller, J. Math. Phys. 22, 1196 (1981).

${ }^{26}$ A. S. Pikovskii, M. I. Rabinovich, and V. Yu. Trakhtengerts, Sov. Phys. JETP 47, 715 (1978). 